\title{
Environmental life-cycle assessment of a military explosive production unit: a preliminary approach
}

\author{
Daniele Costa ${ }^{1}$, Erick Galante ${ }^{2}$, Izabela Andrade ${ }^{3}$, Jéssica Cunha ${ }^{3}$ \\ ${ }^{1}$ Doctoral Program in Environmental Engineering, Faculty of Engineering University of Porto, \\ Porto, Portugal (daniele.costa@fe.up.pt); ${ }^{2}$ Military Institute of Engineering, Rio de Janeiro, \\ Brazil (egalante@ime.eb.br); ${ }^{3}$ Military Institute of Engineering, Rio de Janeiro, Brazil
}

\begin{abstract}
It is generally known that plants of the chemical industry have great potential to generate environmental impacts and health hazards. However, systematic measurements of these impacts are not common. Life Cycle Assessment (LCA) is a tool that allows quantitative environmental impacts evaluation and it was applied in a preliminary approach to analyze selected subsystems in a $\operatorname{RDX}(1,3,5-$ trinitroperhydro-1,3,5-triazine) military production unit. LCA was applied through analysis of the plant data in the free software OpenLCA, using ELCD (European Reference Life Cycle Database) and the TRACI (Tool for the Reduction and Assessment of Chemical and other environmental Impacts) method. As a result, the following impact categories were assessed: acidification, ecotoxicity, eutrophication, global warming, human health, ozone depletion, photochemical oxidation and resource depletion.
\end{abstract}

Subject Headings. Explosive, environmental assessment, impact study Author Keywords. Life Cycle Assessment (LCA), environmental impacts, RDX

\section{Introduction}

Explosives are substances of great importance in human development. Besides their traditional use by the military, explosives are also used in civilian applications, such as construction and natural resources exploitation (e.g. mining and oil exploration). Explosives are chemical substances or mixtures of substances which rapidly react by heating or attrition and generate large volumes of gas and heat.

The objective of this work is to perform a preliminary evaluation of environmental aspects and potential impacts of the RDX production in a Brazilian military facility, through the usage of Life Cycle Assessment (LCA) technique. RDX stands for "royal demolition explosive" and it can also be referred as cyclonite or hexogen. RDX is a white crystalline solid used as an explosive.

LCA is a technique standardized by ISO 14040:2006, which defines it as a "compilation and evaluation of the inputs, outputs and the potential environmental impacts of a product system throughout its life cycle". The methodological framework for LCA consists of four phases, which are: goal and scope definition, inventory analysis, impact assessment and interpretation (ISO 14040:2006).

A life cycle (LC) approach to explosives is not systematically evaluated in literature, which was also reported by Ferreira, Freire, and Ribeiro (2015). This fact reflects an important barrier to reduce the environmental impacts associated to explosive production and usage, fulfillment of legal requirements and implementation of environmental management practices. 
Another relevant goal of this work is to demonstrate the usage of open source LCA software in academic context and to identify its limitations. Currently, the costs of licenses for LCA software represent an important barrier for LCA to become widespread in research and education institutions in developing countries.

This article presents 5 sections. In section 2 a review of military explosives usage in the world, particularly RDX, its usage, fabrication and impacts is presented. In section 3 LCA methodology and facility data are presented and considered. Section 4 is dedicated to the presentation of the life cycle impact assessment ( $L C I A)$ method used. Results and its interpretation are presented in section 5.

\section{Explosives and environment}

The substance named 1,3,5-trinitroperhydro-1,3,5-triazine (IUPAC), hexahydro-1,3,5-trinitro1,3,5-triazine hexogen, or simply $\mathrm{RDX}$ is a military explosive. RDX $\left(\mathrm{C}_{3} \mathrm{H}_{6} \mathrm{~N}_{6} \mathrm{O}_{6}\right)$, whose molecular structure is shown in Figure 1 , is considered as a secondary high explosive, i.e., a compound that presents a fast detonation when initiated by a blow or shock (Deuren et al. 2002).

RDX, TNT (2,4,6-trinitrotoluene), HMX (1,3,5-trini-tro-1,3,5-triazacyclohexane) together are commonly found among contemporary military munitions (Zheng et al. 2009). RDX exposure is associated and primarily limited to the surroundings of ammunition plants and military installations where this product is manipulated, manufactured, converted to munitions, packed, loaded or released through the demilitarization of antiquated munitions (ATSDR 2012).

RDX, TNT and HMX can be considered toxic to humans and other organisms (Zheng et al. 2009). Besides, RDX is very sensitive to electrical sparks, shock, heat and impact, among some other stimuli, which demonstrate the high potential of incidents and impacts when handling this material (Galante et al. 2014, Lynch, Brannon, and Delfino 2002, ATSDR 2012).

RDX exposure may occur by inhalation, ingestion, or dermal absorption and the most probably mode of exposure is by the consumption of contaminated water or the consumption of agricultural crops irrigated with contaminated water (ATSDR 2012). Lynch, Brannon, and Delfino (2002) propose that dissolution into water can be considered the main migration route for nonvolatile explosives and their work has determined the dissolution rates of three high explosives: RDX, HMX and TNT.

Military explosives contamination is widely discussed in literature (Agus and Hassan 2011, Alavi et al. 2011, Best et al. 1999, Lynch, Brannon, and Delfino 2002, Zheng et al. 2009). Despite the fact that RDX's environmental impacts are referred in literature in several articles, the majority of them only consider punctual treatment technology methodologies (such as Vila et al. (2007), Vila, Lorber-Pascal, and Laurent (2007) and Chen et al. (2011), among others) and do not present a comprehensive and systematic life cycle perspective, not allowing the identification of impacts at each stage of explosive existence (i.e., from its "cradle to gate").

Other relevant papers have detailed explosives behavior in environment. Zheng et al. (2009) have analyzed the transport and decomposition of RDX, HMX and TNT explosives and their metabolites in sediments. Alavi et al. (2011) have analyzed the fate and transport of for unexploded ordnance chemicals (RDX, HMX, TNT and DNT, 2,4-dinitrotoluene) in volcanic soils and have also evaluate the potential risk of groundwater contamination associated to these chemicals. 
The treatment and behaviour analysis are the more frequent approaches in papers regarding explosives and this fact can be assumed to be a consequence of the fact that many US military and defense installations have soil, sediment, surface water, and ground water contaminated with explosives (Pennington and Brannon 2002). However, a comprehensive full assessment approach of its environmental impacts is scarce in literature.

Ferreira, Freire, and Ribeiro (2015) have conducted a study in which they performed the life cycle assessment (LCA) of a civil explosive. They also report the scarcity of other papers referring to the LCA of explosives. Kellenberger et al. (2007) is the only work that presents similarity with the goals in Ferreira, Freire, and Ribeiro (2015) and of the ones aimed by the present study.

In the plant assessed by the present paper, RDX is produced by nitrating hexamethylaminetriamine ( $66 \mathrm{H} 12 \mathrm{~N} 4)$ using concentrated nitric acid (above $90 \mathrm{wt} . \%)$. The later precipitation of the explosive occurs by adding cold water. The industrial manufacture of RDX used for this study adapts the process patented by William J. Lukasavage, Steven Nicolich, and Slagg (1992) and Meredith (1974).

The process in the unit under study can be summarized into the following stages: hexamine nitration, precipitation, purification, coaching, drying, grading and packing. At the same time, nitrous vapors absorptions, raw materials recovery of and wastewater treatment are performed. The key features of the manufactory process, studied in section 4 of this work, are presented in Figure 1. The equipment flowchart consists on mixture in nitration vessels, boilers, followed by filtration of the final product.

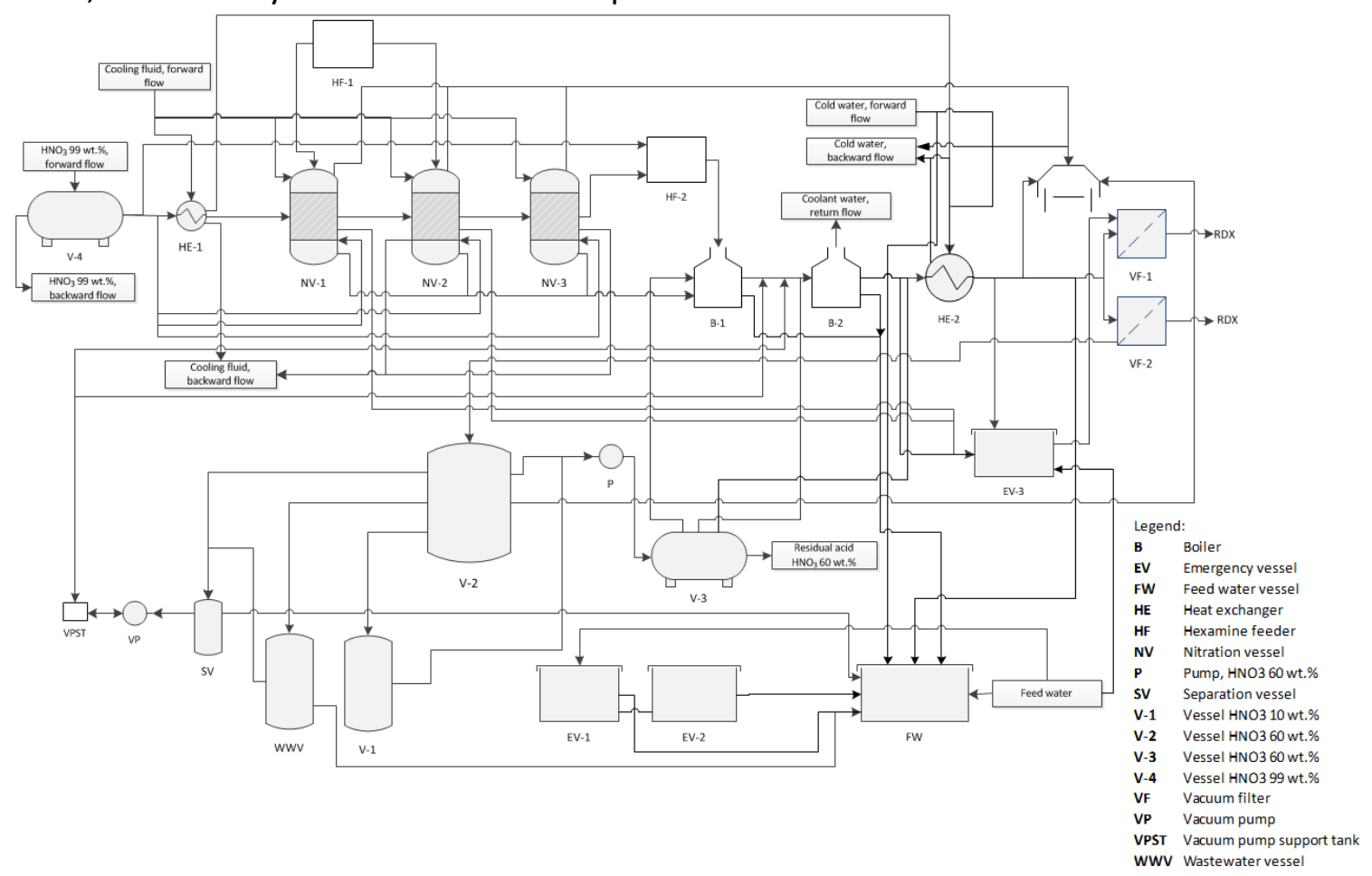

Figure 1: Process Diagram to manufacture RDX

Conducting LCA for a RDX manufacture facility is relevant since it may allow the determination of possible routes of exposure to this product. An adequate LCA for RDX has also the potential to support its risk assessment in manipulation sites, including occupational exposure, allowing linking contamination sources, transmission pathways, and exposure potential. 


\section{Life-cycle assessment and RDX production inventory}

In this work, a comprehensive Life-Cycle Assessment for RDX production was conducted in a Brazilian military facility that produces explosives. The evaluation of LC, through the lifeCycle Assessment (LCA) methodology aims to identify and quantify impacts in explosive production, creating awareness of environmental aspects linked to the process.

The goal and scope of the present study is to identify and quantify the most relevant impacts in selected production processes in a facility that produces RDX, a military explosive. To achieve these marks, the compilation of the life cycle inventory and the life cycle impact assessment were performed with openLCA software and the European Reference Life Cycle Database (ELCD).

Currently, different LCA software products are available in the market. However, the choice for using free software relies on the need to make LCA affordable for academic usage and also to promote LCA in engineering education.

The subsystems analyzed in this preliminary LCA were chosen considering the mass and energy balances, as well as any potential hazards to the environment. The subsystems chosen were hexamine nitration, nitrous vapors recovery and filtration of RDX; they are described in subsections 3.2 to 3.4. System's boundaries of the studied plant (briefly described in section 0) are exhibited in Figure 2, which highlights the subsystems that were analyzed.

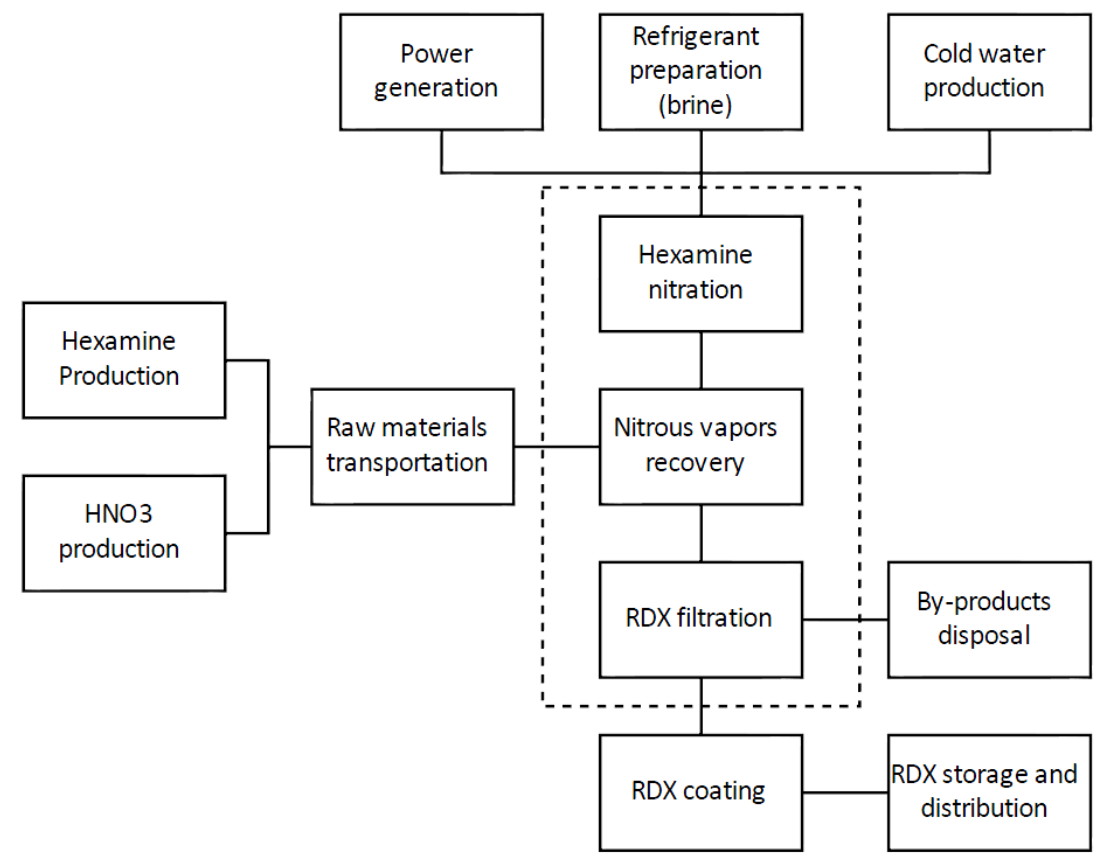

Figure 2: System boundaries

These subsystems were selected considering the independence of the stages and dynamic differences in the processes, as well as considering that any chemical reaction in the entire process occurs only within these subsystems, while the other processes are mainly physical transformation and support stages. Hexamine nitration is done in batch mode while the recovery of nitrous vapors and filtration of RDX are continuous processes.

In subsections 3.1 to 3.4 , the available manufacturing data are presented and input and output data for each subsystem are inventoried. These subsections consist on the life cycle inventory analysis ( $\mathrm{LCl}$ ) of RDX manufacturing and together they represent the step two required for a LCA study. 


\subsection{LCA general data and limitations}

Energy consumption during RDX production is associated to fuel oil in the boilers and public electricity. No significant inputs are used to maintain temperature conditions at the plant. The water supply comes from a wheel within the facility. Hexamine and nitric acid at $99 \%$ wt.\% are the main raw materials used in the process.

The majority of the data used in this paper is controlled and given by the manufacturer, although the water consumption had to be estimated. The energy charges represent equipment consumption in the site, leading to the consideration of all drops in both pressure and heat during the process.

It was considered that hexamine reacts at $96 \%$ (the yield factor for the facility under study ranges between 94 and 98\%), following the chemical reactions ( 1 and 2 ). It is considered that the remaining hexamine is consumed by the secondary reaction, according to Equation 2.

$$
\begin{aligned}
\left(\mathrm{CH}_{2}\right)_{6} \mathrm{~N}_{4}+4 \mathrm{HNO}_{3} & \rightarrow \mathrm{C}_{3} \mathrm{H}_{6} \mathrm{~N}_{6} \mathrm{O}_{6}+3 \mathrm{HCOH}+\mathrm{NH}_{4} \mathrm{NO}_{3} \\
\left(\mathrm{CH}_{2}\right)_{6} \mathrm{~N}_{4}+6 \mathrm{HNO}_{3} & \rightarrow \mathrm{C}_{3} \mathrm{H}_{6} \mathrm{~N}_{6} \mathrm{O}_{6}+6 \mathrm{H}_{2} \mathrm{O}+3 \mathrm{CO}_{2}+2 \mathrm{~N}_{2}
\end{aligned}
$$

In the studied plant, the bulk temperature is maintained between $12^{\circ} \mathrm{C}$ and $15^{\circ} \mathrm{C}$ during hexamine addition into nitric acid. The exothermical reaction is allowed to go up to $18^{\circ} \mathrm{C}$, temperature in which it is dumped into quench tanks due to safety concerns. After nitration, the mixture is heated (via steam) to $60^{\circ} \mathrm{C}$ for 60 minutes, then the mixture is cooled and the RDX precipitate is filtered, washed and dried. Each stage (nitration, heating and cooling) is held in a different vessel.

\subsection{Nitration of hexamine}

The nitration of hexamine subsystem uses hexamine, concentrated nitric acid, refrigerant fluid (brine), water and energy as inputs. As a result of the process, the outputs are RDX and other by-products as well as the return of coolant solution, nitrous vapor and heat.

The analyzed plant uses 300 liters of $90 \mathrm{wt} . \%$ acetic acid for the production of $50 \mathrm{~kg}$ of RDX for each 2 hours per batch. Considering all data presented and the mass balances, as well as the stoichiometry of the reaction, it was possible to determine the nitration of hexamine subsystem mass balance, which is presented in Table 1.

\begin{tabular}{ccc}
\multicolumn{2}{c}{ Input $(\mathbf{k g} / \mathbf{h})$} & Output $(\mathrm{kg} / \mathrm{h})$ \\
\hline Hexamine & 16.44 & 0 \\
HNO3 & 220.5 & 172.64 \\
RDX & 0 & 25 \\
H2O & 4.5 & 11.84 \\
NH4NO3 & 0 & 4.50 \\
HCOOH & 0 & 9.06 \\
CO2 & 0 & 7.43 \\
N2 & 0 & 3.15 \\
NH3 & 0 & 0.32 \\
NO & 0 & 2.95 \\
NO2 & 0 & 4.53 \\
\hline
\end{tabular}

Table 1: Mass balance

Regarding energy, it was considered energy for operating the compressor that keeps the cooling fluid supplied to the nitration vessel at $-18^{\circ} \mathrm{C}$. The heating system uses steam generated in a boiler that burns fuel (heavy oil), hence the heat is measured in terms of fuel consumption. The amount of water required for operating the boiler in the considered 
conditions was calculated. When considered together, these data lead to Table 2, which presents the inputs of this subsystem, and Table 3, which presents its outputs.

\begin{tabular}{ccc} 
& Variable & Input (kg/h) \\
\hline Reagents & Hexamine & 16.44 \\
& Nitric acid, 98 wt.\% & 225 \\
& $\begin{array}{c}\text { Electricity to operate } \\
\text { refrigeration (via gas } \\
\text { Energy }\end{array}$ & 26.11 \\
& $\begin{array}{c}\text { compression) } \\
\text { Boiler fuel } \\
\text { (BPF oil) }\end{array}$ & \\
Elementary & Coolant fluid & 75 \\
fluxes & Steam & 0 \\
\hline
\end{tabular}

Table 2: Inputs - nitration of hexamine

\begin{tabular}{lcc} 
& Variable & Output $(\mathrm{kg} / \mathrm{h})$ \\
\hline \multirow{2}{*}{ Products } & RDX & 25 \\
& Nitrous vapors & 15.46 \\
\multirow{2}{*}{ Excesses } & Nitric acid, 50 wt.\% & 334.5 \\
& HMX & 0 \\
\hline
\end{tabular}

Table 3: Outputs - nitration of hexamine

\subsection{Recovery of nitrous vapors}

Nitrous vapors generated by hexamine nitration proceed to a recovery unit as a nitric acid solution $50 \mathrm{wt} \%$ In this site, the nitrous oxide stream is washed with water in a countercurrent flow is used to wash this stream.

It was assumed that all the NOx generated in the nitration is converted to nitric acid 50 wt.\% within a recovery tower. This recovery tower makes the NOx flow upstream against a column of water, therefore recovering nitric acid at the bottom. The actual values obtained by the plant operator in the facility ranges from $40 \mathrm{wt} . \%$ to $60 \mathrm{wt} . \%$, so an average value was considered. This assumption should not impact the overall results, since this should be the actual result from one year of operation. The recovered nitric acid obtained in this process sold by the facility, hence no recycle is considered under the analysis.

This subsystem has nitrous vapors, energy and water as inputs, which after reaction produces residual nitric acid at $50 \mathrm{wt} . \%$. Other outputs of the process are wash water and heat. The energy necessary in this stage is considered as an elementary flux. Inputs and outputs of this subsystem are summarized in Table 4 and Table 5.

\begin{tabular}{ccc}
\multicolumn{2}{c}{ Variable } & Input $(\mathrm{kg} / \mathrm{h})$ \\
\hline Reagent & $\begin{array}{c}\text { Nitrous vapors } \\
\text { In steam }\end{array}$ & 15.46 \\
Energy & $\begin{array}{c}\text { equivalent } \\
\text { Water }\end{array}$ & 2.98 \\
Elementary flux & Water
\end{tabular}

Table 4: Inputs - Recovery of nitrous vapors

\begin{tabular}{ccc} 
& Variable & $\begin{array}{c}\text { Output } \\
(\mathbf{k g} / \mathbf{h})\end{array}$ \\
\hline Product & Nitric acid (50 wt.\%) & 30.92 \\
Excess & Washing water & 84.54 \\
\hline \multicolumn{2}{c}{ Table 5: Outputs - Recovery of nitrous vapors }
\end{tabular}

\subsection{Filtration of RDX}

Filtration of RDX is a subsystem that produces pure RDX. Its inputs are RDX (as a water solution). The power and water while the output is residual nitric acid around $60 \% \mathrm{wt.} \%$, 
considering that filtration is performed at $100 \%$ efficiency (which is verified in the facility by assessing the RDX content in the wastewater). The amount of water required during this process was estimated considering attainment of an excess of nitric acid at $30 \mathrm{wt} . \%$. Considering the given electric energy consumption, Table 6 and Table 7 present, respectively, the inputs and outputs associated to this subsystem.

\begin{tabular}{ccc} 
& Variable & Input (kg/h) \\
\hline Entrances & $\begin{array}{c}\text { RDX with impurity } \\
\text { Fresh water }\end{array}$ & 378.56 \\
Elementary flux & $\begin{array}{c}\text { Electricity to operate } \\
\text { refrigeration (via gas } \\
\text { compression) }\end{array}$ & 2.98 \\
& 2.98 \\
\hline
\end{tabular}

Table 6: Input - filtration of RDX

\begin{tabular}{ccc} 
& Variable & Output $(\mathrm{kg} / \mathrm{h})$ \\
\hline Product & RDX & 25 \\
\hline Excess & Residual acid, 30 wt.\% & 575.47 \\
\hline \multicolumn{3}{c}{ Table 7: Output - filtration of RDX }
\end{tabular}

\section{Results}

The environment indicator method for conducting the third LCA step, LCIA, was the Tool for the Reduction and Assessment of Chemical and other environmental Impacts (TRACI). This choice was made considering the toxicity measures of this method, as well as its usage allows comparison with other explosive units in which TRACl has been used as an indicator.

Operational conditions of the site are mild, with no extreme pressure and temperature conditions. However, the wastewater generated in the site has a considerable concentration of nitric acid (around $60 \mathrm{wt} . \%$ ) and cannot be discharged without treatment. The operational decision is to collect and sell this wastewater in the form of weak nitric acid as raw material for other manufacturers. Table 8 presents the main results calculated considering TRACI impact categories, considering data from ELCD.

\begin{tabular}{cc} 
Impact category & Total value \\
\hline Acidification (kgSO 2 eq) & 4.60 \\
Ecotoxicity (CTUe) & $8.57 \mathrm{E}-03$ \\
Eutrophication (kgNeq) & 2.04 \\
Global Warming (kgCO $2 \mathrm{eq})$ & $1.07 \mathrm{E}-01$ \\
Human Health - carcinogenics(CTUh) & $1.25 \mathrm{E}-11$ \\
Human Health - non-carcinogenics(CTUh) & $3.34 \mathrm{E}-12$ \\
Human Health - air pollutants criteria (kg 2,5PMeq) & $3.51 \mathrm{E}-04$ \\
Ozone depletion (kgCFC-11eq) & $1.92 \mathrm{E}-08$ \\
Photochemical oxidation (gO 3 eq) & $4.05 \mathrm{E}-02$ \\
Resource depletion - Fossil fuels (MJ surplus) & $1.90 \mathrm{E}-02$ \\
\hline
\end{tabular}

Table 8: LCA results

Analyzing the data presented in tables 1 to 7, one can compare the results for each subsystem. The comparison among the selected subsystems has demonstrated that hexamine nitration is the most relevant process for the ten environmental impact categories analyzed. This subsystem has a participation of more than $90 \%$ in all impacts categories analyzed.

In the future, a wider LCA of the RDX should be conducted through the evaluation of more stages of the production process, guaranteeing a full evaluation of its manufacturing. In addition to that, raw materials and final products transportation data should be taken into 
consideration for a more complete LCA. Considering these data would significantly improve the analysis and generate more adequate results.

Another suggestion for future research is to consider different LCIA methods. The purpose of this is to compare methods and obtain more robust conclusions in the RDX manufacturing impacts, as performed by Ferreira, Freire, and Ribeiro (2015). It is also suggested to adopt different LCIA methods that would allow a better understanding of the differences between methods and their adequacy.

\section{Conclusions}

This paper has developed a preliminary LCA for RDX production in a military explosive unit. Three subsystem of the process were selected and the results demonstrated that the main contributor for the ten impact categories analyzed is the hexamine nitration subsystem.

The lack of more accurate data could not allow a full LCA of the RDX production at plant. Moreover, maybe this work is the first LCA in an explosive plant in Brazil and as so, it may be a first reference for similar future studies.

Despite ELCD usage does not invalidate the study, the usage of a national database would provide more precise results. As a consequence, the usage of these data would allow a best correlation to reality, since ELCD has important limitations for explosive assessment.

For more accurate results, new studies in this plant should also consider distances of raw materials and final destination of the products whenever it is possible. In order to develop a complete LCA in the plant, future studies may analyze more subsystems for a complete assessment of the RDX production unit.

Moreover, explosive manufacturing have a very high potential for hazardous outcomes in case a process upset occurs. Despite that, manufacture, testing, sale, storage, and transportation of explosives also require special consideration and in this perspective, a LCA study may be seen as a relevant part of risk assessment of these products.

\section{References}

Agus, Arawati, and Za'faran Hassan. 2011. "Enhancing Production Performance and Customer Performance Through Total Quality Management (TQM): Strategies For Competitive Advantage." Procedia - Social and Behavioral Sciences no. 24:1650-1662. DOI: 10.1016/j.sbspro.2011.09.019.

Alavi, G., M. Chung, J. Lichwa, M. D'Alessio, and C. Ray. 2011. "The fate and transport of RDX, HMX, TNT and DNT in the volcanic soils of Hawaii: a laboratory and modeling study." J Hazard Mater no. 185 (2-3):1600-4. DOI: 10.1016/j.jhazmat.2010.10.039.

ATSDR, US Agency for Toxic Substances and Disease Registry. 2012. ToxGuide ${ }^{\mathrm{TM}}$ for RDX C3H6N606. USA.

Best, Elly P. H., Susan L. Sprecher, Steven L. Larson, Herb L. Fredrickson, and Bader F. Darlene. 1999. "Environmental behavior of explosives in groundwater in groundwater from the Milan army ammunition plant in aquatic and wetland plant treatments. Removal, mass balances and fate in groundwater of TNT and RDX." Chemosphere no. 38 (14):3383-3396. DOI: 10.1016/S0045-6535(98)00550-5.

Chen, Yong, Lei Hong, Weiqing Han, Lianjun Wang, Xiuyun Sun, and Jiansheng Li. 2011. "Treatment of high explosive production wastewater containing RDX by combined electrocatalytic reaction and anoxic-oxic biodegradation." Chemical Engineering Journal no. 168 (3):1256-1262. DOI: 10.1016/j.cej.2011.02.032. 
Deuren, Julie Van, Teressa Lloyd, Shobha Chhetry, Raycharn Liou, and James Peck. 2014. Remediation Technologies Screening Matrix and Reference Guide, 4th Edition 2002 [cited December 2014]. Available from http://www.frtr.gov/matrix2/section2/2_10_1.html.

Ferreira, Carlos, Fausto Freire, and José Ribeiro. 2015. "Life-cycle assessment of a civil explosive." Journal of Cleaner Production no. 89:159-164. DOI: 10.1016/j.jclepro.2014.11.027.

Galante, Erick B. F., Daniele M. B. da Costa, Assed N. Haddad, and Isaac José A. L. dos Santos. 2014. "Risk Assessment for Hexamine Nitration into RDX." Journal of Aerospace Technology and Management no. 6 (4):373-388. DOI: 10.5028/jatm.v6i4.380.

ISO 14040:2006, International Organization for Standardization. Environmental management - Life cycle assessment - Principles and framework. In ISO 14040:2006.

Kellenberger, D., Althaus H.-J., N Jungbluth, and T Künniger. 2007. Life Cycle Inventories of Building Products. In Final report ecoinvent data v2.0 No. 7. Dübendorf, $\mathrm{CH}$.

Lynch, Jason C., James M. Brannon, and Joseph J. Delfino. 2002. "Dissolution rates of three high explosive compounds: TNT, RDX, and HMX." Chemosphere no. 47:725-734. DOI: 10.1016/S0045-6535(02)00035-8.

Meredith, Joseph A. 1974. Preparation of RDX. United States of America: The United States of America as represented by the Secretary of the Army.

Pennington, Judith C., and James M. Brannon. 2002. "Environmental fate of explosives." Thermochimica Acta no. 384 (1-2):163-172. DOI: 10.1016/S0040-6031(01)00801-2.

Vila, M., S. Lorber-Pascal, and F. Laurent. 2007. "Fate of RDX and TNT in agronomic plants." Environ Pollut no. 148:148-154. DOI: 10.1016/j.envpol.2006.10.030.

Vila, M., S. Mehier, S. Lorber-Pascal, and F. Laurent. 2007. "Phytotoxicity to and uptake of RDX by rice." Environmental Pollution no. 145 (3):813-817. DOI: 10.1016/j.envpol.2006.05.009.

William J. Lukasavage, Steven Nicolich, and Norman Slagg. 1992. Process for preparation of RDX. United States of America: The United States of America as represented by the Secretary of the Army (Washington, DC).

Zheng, W., J. Lichwa, M. D'Alessio, and C. Ray. 2009. "Fate and transport of TNT, RDX, and HMX in streambed sediments: Implications for riverbank filtration." Chemosphere no. 76 (9):1167-77. DOI: 10.1016/j.chemosphere.2009.06.043. 